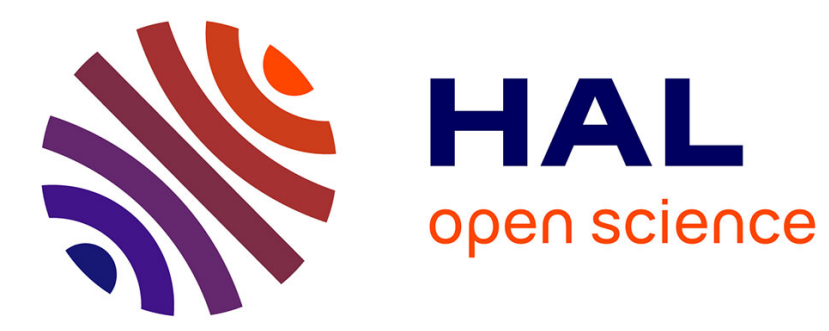

\title{
Integrated Smart Energy System Based on Production-Oriented Consumption
}

\author{
Ivan Popov, Alexander Krylatov, Victor Zakharov
}

\section{To cite this version:}

Ivan Popov, Alexander Krylatov, Victor Zakharov. Integrated Smart Energy System Based on Production-Oriented Consumption. 17th Working Conference on Virtual Enterprises (PRO-VE), Oct 2016, Porto, Portugal. pp.265-273, 10.1007/978-3-319-45390-3_23 . hal-01614581

\section{HAL Id: hal-01614581 \\ https://hal.inria.fr/hal-01614581}

Submitted on 11 Oct 2017

HAL is a multi-disciplinary open access archive for the deposit and dissemination of scientific research documents, whether they are published or not. The documents may come from teaching and research institutions in France or abroad, or from public or private research centers.
L'archive ouverte pluridisciplinaire HAL, est destinée au dépôt et à la diffusion de documents scientifiques de niveau recherche, publiés ou non, émanant des établissements d'enseignement et de recherche français ou étrangers, des laboratoires publics ou privés. 


\title{
Integrated Smart Energy System Based on Production- Oriented Consumption
}

\author{
Ivan Popov ${ }^{1}$, Alexander Krylatov ${ }^{2,3}$ and Victor Zakharov ${ }^{2,3}$ \\ ${ }^{1}$ Control Methods and Robotics Lab, TU Darmstadt, Germany, \\ ipopov@rmr.tu-darmstadt.de \\ ${ }^{2}$ Saint Petersburg State University, 7/9 Universitetskaya nab., \\ St. Petersburg, 199034 Russia \\ a.krylatov@spbu.ru, v.zaharov@spbu.ru \\ ${ }^{3}$ Solomenko Institute of Transport Problems of the Russian Academy of Sciences, \\ 13 12-th Line VO, St. Petersburg, 199178 Russia, \\ info@iptran.ru
}

\begin{abstract}
Modern power grids are facing a number of challenges, such as everincreasing consumption, development of alternative energy generators, and decentralization of energy markets. Renewable energy sources are strongly weather-dependent and, therefore, cannot provide reliable production profiles. This leads us to the concept of demand side management, where energy users modify their consumption patterns due to the availability of generation capacities. Additionally, power flow distribution should be carefully studied in order to avoid overloads in the grid. In this work, we describe a multi-supplier multiconsumer grid model and formulate a problem of an optimal energy contract assignment with respect to power flow constraints. Though generally energy consumers are selfish, we assume their readiness for collaboration within a smart energy system. We offer a cooperative solution for this problem on the assumption of appropriate coordination between agents. Finally, we provide an example illustrating the applicability of our methods.
\end{abstract}

Keywords: demand side management, multi-agent systems, power grids, congestion, load flow.

\section{Introduction}

The structure of power supply has been changing significantly in last two decades. A new vision of power grid structures has appeared due to set of reasons, firstly, such as ever-growing energy consumption and development of alternative energy sources. New requirements for power grid structures with generators of renewable energy exacerbate such a crucial problem in power grid management as imbalance between energy demand and supply. Hence, the power system should turn to the production-oriented consumption that leads us to the idea of demand side management (DSM).

Much work has been done in the field of demand side management and, more specifically, demand response. Basic pricing mechanisms for systems with single supplier and multiple consumers are studied by [1], [2]. Distributed generation and 
storage are considered in [3], [4]. Coalition formation for local networks is examined in various scenarios in [5], [6] with use of methods of cooperative game theory. However, the questions of power grid's topology and congestion in transmission lines are not taken into account in these works. In present paper we fill this gap since transmission network is a significant element of any power grid system.

Actually, the question of collaboration in energy market has long been irrelevant, and, in contrast to smart grid consumer collaboration, competitive market forces of power generation, consumption, and transmission have been deeply investigated [7], [8], [9]. Nowadays, the newly established circumstances force us to investigate collaborative mechanisms in power consumption. Due to modern technologies, generators and consumers are now able to be integrated spatially in a smart local (nodal) power system. Smart grid power system could guarantee the conditions of optimal nodal demand-side consumption by virtue of the agreement between consumers to collaborate in energy consumption.

In this paper we formulate a model of cooperating consumers in a power network. We show that an integrated smart energy system could reduce overall costs in congested networks with multiple producers. An example of a network with tree structure is considered in order to show the applicability of this idea.

\section{Model Description}

In this section, we formulate the power grid model with multiple generators and consumers. Each generator has a production cost function, and it depends on the total amount of energy this generator is assigned to produce. Consumers are price-taking agents with energy demand that they need to meet. Both production and transmission costs are covered by consumers. We now discuss all elements of this model in detail.

\subsection{Network Structure}

Consider a directed connected graph $G=(V, A)$, where $V$ is a set of nodes and $A$ is a set of arcs. Since energy current in a power grid can flow in either direction of any link, we assume that for any $\operatorname{arc}(k, l) \in A$ there is also an $\operatorname{arc}(l, k) \in A$. We also claim that only one of these two arcs can be used at the same time (i.e., energy current cannot proceed in both directions of a link simultaneously).

We enumerate nodes in $V$ a specific way, so that set $V$ consists of three subsets: a set of $m$ consumer nodes $V_{Q}=\{1, \ldots, m\}$, a set of $n$ producer nodes $V_{P}=\{m+1, \ldots, m+n\}$, and a set of all other nodes $V_{O}=\{m+n+1, \ldots,|V|\}$.

Consumers make bilateral energy contracts with multiple producers in order to meet their energy demands. By $e_{i j} \geq 0$ we denote a contract between a consumer $i \in V_{Q}$ and a producer $j \in V_{P}$, and $d_{i} \leq 0$ is an energy demand of consumer $i \in V_{Q}$. Let us also define $\bar{e}_{i}=\left\{e_{i j}, j \in V_{P}\right\}$, consumer $i$ 's contract profile, and $\bar{e}=\left\{e_{i j}, i \in V_{Q}, j \in V_{P}\right\}$, a 
contract profile of all consumers. Therefore, demand constraints take the following form:

$$
d_{i}+\sum_{j=m+1}^{m+n} e_{i j}=0, \quad i \in V_{Q} .
$$

For all other nodes $k \in V_{P} \cup V_{O}$, we also define $d_{k}$ :

$$
\begin{gathered}
d_{k}=\sum_{i=1}^{m} e_{i k}, \quad k \in V_{P}, \\
d_{k}=0, \quad k \in V_{O} .
\end{gathered}
$$

Let us call $d_{k}$ an energy input in a node $k \in V$. It is positive in producer nodes, negative in consumer nodes, and equal zero otherwise.

For each producer $j \in V_{P}$ we define a unit production $\operatorname{cost} \alpha_{j}\left(d_{j}\right) \geq 0$, a function of an energy input $d_{j}$, the total sum of contracts assigned to this producer. Consumers share production costs proportionally to their contracts, and for a given contract profile $\bar{e}$ consumer $i \in V_{Q}$ pays to producer $j \in V_{P}$ the following price:

$$
c_{i j}(\bar{e})=e_{i j} \cdot \alpha_{j}\left(d_{j}\right)=e_{i j} \cdot \alpha_{j}\left(\sum_{i=1}^{m} e_{i j}\right) .
$$

Therefore, we can determine the overall production cost of each consumer $i \in V_{Q}$ for a given contract profile $\bar{e}$ :

$$
c_{i}(\bar{e})=\sum_{j \in V_{P}} c_{i j}(\bar{e})=\sum_{j \in V_{P}} e_{i j} \cdot \alpha_{j}\left(\sum_{i=1}^{m} e_{i j}\right) .
$$

\subsection{Load Flow}

Transmission costs are the second type of expenses covered by consumers. These costs depend on the load flow in a network, and we require to make several further definitions. By power flow $f_{k l} \geq 0$ in $\operatorname{arc}(k, l) \in A$ we denote a non-negative value of current flowing in this arc. According to our assumption, $f_{k l}$ and $f_{l k}$ cannot be both positive. A flow profile $\bar{f}=\left\{f_{k l},(k, l) \in A\right\}$ is a set of flows in all arcs. We assume that each arc $(k, l) \in A$ charges a transmission fee $\beta_{k l}\left(f_{k l}\right)$ that must be shared between consumers using this arc.

In order to determine the transmission cost of consumer $i$ similarly to (4), we need to answer two questions: how does flow profile $\bar{f}$ depend on contract profile $\bar{e}$ ? And how should the transmission costs be shared between consumers? 
The first question can be answered by applying Kirchhoff's laws to this power grid. Let us denote all neighbours of node $k$ by $W_{k}=\{l \in V \mid(k, l) \in A\}$. Then the first Kirchhoff's law takes the following form:

$$
\sum_{l \in W_{k}} f_{k l}-\sum_{l \in W_{k}} f_{l k}=d_{k}, \quad \forall k \in V .
$$

If $\Theta_{k l}\left(f_{k l}\right)$ is a voltage change function for an $\operatorname{arc}(k, l) \in A$, and $\pi_{k}$ is a potential in node $k \in V$, we can formulate the second Kirchhoff's law for our model:

$$
\pi_{k}-\pi_{l}=\Theta_{k l}\left(f_{k l}\right), \quad \forall(k, l) \in A .
$$

It is well-known that the flow profile can be found as a solution of a non-linear optimization problem (cf. Ch. 2.6 in [10]):

$$
\min _{\bar{f}} \sum_{(k, l) \in A} \int_{0}^{f_{k l}} \Theta_{k l}(s) d s,
$$

subject to

$$
\begin{gathered}
\sum_{l \in W_{k}} f_{k l}-\sum_{l \in W_{k}} f_{l k}=d_{k}, \quad \forall k \in V, \\
f_{l k} \geq 0, \quad \forall(k, l) \in A,
\end{gathered}
$$

where contract profile $\bar{e}$ defines coefficients $\left\{d_{k}\right\}$ in (8).

By $\bar{f}(\bar{e})$ we denote the solution of problem (7)-(9) for a given $\bar{e}$. Mathematical programming formulation of the problem of finding equilibrium currents and voltages in electrical networks is an electrical network analogy of traffic equilibrium problem [10], [11], [12].

The second question is more complicated, since electricity is a homogeneous product, and unlike transportation problems it is not possible to track what portion of energy belongs to which consumer-producer contract. There are different methods used to determine transmission cost pricing, e.g. [13]. In general case, we define a set of functions $\left\{\delta_{i}^{k l}(\bar{e})\right\}$ that impose the cost sharing rule:

$$
\begin{gathered}
\sum_{i \in V_{Q}} \delta_{i}^{k l}(\bar{e})=1, \forall(k, l) \in A, \\
\delta_{i}^{k l}(\bar{e}) \geq 0, \quad \forall(k, l) \in A, \quad \forall i \in V_{Q} .
\end{gathered}
$$

According to this rule, one can find transmission cost of consumer $i \in V_{Q}$ charged by $\operatorname{arc}(k, l) \in A$ as follows:

$$
t_{i}^{k l}(\bar{e})=\delta_{i}^{k l}(\bar{e}) \cdot \beta_{k l}\left(f_{k l}(\bar{e})\right) .
$$

Thus, total transmission cost paid by consumer $i$ is 


$$
t_{i}(\bar{e})=\sum_{(k, l) \in A} t_{i}^{k l}(\bar{e})=\sum_{(k, l) \in A} \delta_{i}^{k l}(\bar{e}) \cdot \beta_{k l}\left(f_{k l}(\bar{e})\right)
$$

We are ready to formulate a network optimization problem for a system of cooperative consumers.

\section{Integrated Smart Energy System}

Usually, the participants of energy market are assumed to be selfish and independent, that leads us to a system formulated in terms of competitive games. However, if energy resources are scarce and/or uncontrollable (such as renewable energy generation) it may be more profitable for consumers to cooperate. This section formulates a total cost minimization problem for a power grid with multiple suppliers.

\subsection{Total Cost Minimization Problem}

In order to reduce total costs for energy production and transmission, we need to solve a total cost minimization problem. Let us consider the total cost of all consumers, according to (4), (12):

$$
\begin{gathered}
C(\bar{e})=\sum_{i \in V_{Q}}\left(c_{i}(\bar{e})+t_{i}(\bar{e})\right)= \\
\sum_{i \in V_{Q}} \sum_{j \in V_{P}} e_{i j} \cdot \alpha_{j}\left(\sum_{i=1}^{m} e_{i j}\right)+\sum_{i \in V_{Q}} \sum_{(k, l) \in A} \delta_{i}^{k l}(\bar{e}) \cdot \beta_{k l}\left(f_{k l}(\bar{e})\right)= \\
\sum_{j \in V_{P}} d_{j}(\bar{e}) \cdot \alpha_{j}\left(d_{j}(\bar{e})\right)+\sum_{(k, l) \in A} \beta_{k l}\left(f_{k l}(\bar{e})\right) .
\end{gathered}
$$

The minimization problem can be formulated in the following form:

$$
\min _{\bar{e}} \sum_{j \in V_{P}} d_{j}(\bar{e}) \cdot \alpha_{j}\left(d_{j}(\bar{e})\right)+\sum_{(k, l) \in A} \beta_{k l}\left(f_{k l}(\bar{e})\right)
$$

subject to (1), (2),

where flow profile $\bar{f}(\bar{e})$ is found as a solution of optimization problem (7)-(9).

Note that the solution of constrained optimization problem (14), (15) does exist. However, it is not a trivial task to define the uniqueness of the solution in general case. Nevertheless, the properties of a solution could be established via differentiating Lagrangian with respect to $e_{i j}$ and using Kuhn-Tucker conditions. The only thing to be state is that Kuhn-Tucker conditions are necessary and sufficient when product $d_{j}(\bar{e}) \cdot \alpha_{j}\left(d_{j}(\bar{e})\right)$ and $\beta_{k l}\left(f_{k l}(\bar{e})\right)$ are convex functions. Generally, one could use most appropriate powerful numerical method for constrained nonlinear optimization [14]. 


\subsection{Consumer Cooperation}

There are different ways of fair cost sharing among consumers. Ideas of the cooperative game theory may be applied, such as the concept of Shapley value for a coalition of consumers (see, e.g., [15]). Another simplified policy to encourage the cooperation between consumers is to share the total cost proportionally to the demand of each consumer:

$$
C_{i}(\bar{e})=d_{i} \cdot C(\bar{e}), \forall i \in V_{Q} .
$$

In this case each consumer $i$ minimizes the same function (up to a constant coefficient $\left\{d_{i}\right\}$.

In the following example we do not consider any specific rule of cost sharing, but rather the possible total cost reduction in the network without cycles.

\section{Example and Simulation Results}

This section shows how our methods can be applied to a specific example of a network with tree structure.

\subsection{Example}

Consider a network with 7 nodes that is depicted in Figure 1. There are 3 consumers (red nodes), 3 producers (green nodes) and one intermediate node. Therefore, $V_{Q}=\{1,2,3\}, V_{P}=\{4,5,6\}$, and $V_{O}=\{7\}$.

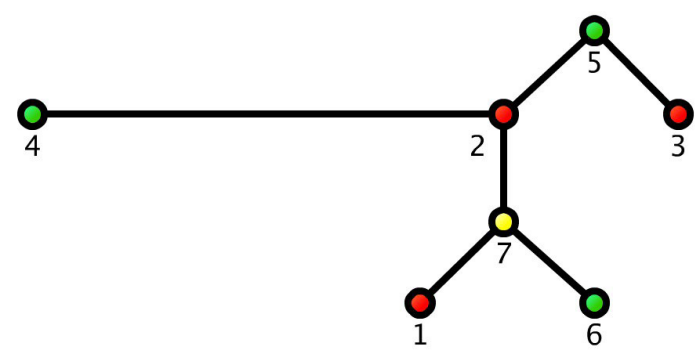

Fig. 1. 7-node network with no cycles

All nodes are located in the same local area except for node 4 that depicts a conventional energy generator, e.g. a power plant. Hence, arc $(4,2)$ is longer than all other arcs, and transmission costs are higher for this arc.

Since there are no cycles in the network, we only need to check the first Kirchhoff's law (5). A flow on each arc is a linear combination of $\left\{e_{i j}\right\}$ :

$$
\hat{f}_{25}=e_{34}+e_{36}-e_{15}-e_{25}, \quad \hat{f}_{53}=e_{34}+e_{35}+e_{36}, \quad \hat{f}_{42}=e_{14}+e_{24}+e_{34},
$$




$$
\hat{f}_{27}=e_{14}+e_{15}-e_{26}-e_{36}, \hat{f}_{71}=e_{14}+e_{15}+e_{16}, \hat{f}_{67}=e_{16}+e_{26}+e_{36} .
$$

The direction of flow in arcs $(2,5)$ and $(2,7)$ may differ depending on the values $\left\{e_{i j}\right\}$. If $\hat{f}_{25}<0$, we assign $\hat{f}_{25}=0$ and $\hat{f}_{52}=-\hat{f}_{25}$. The same is true for $\hat{f}_{27}$.

Let us assume that functions $\left\{\alpha_{j}(\cdot)\right\}$ and $\left\{\alpha_{j}().\right\}$ have the following form:

$$
\begin{gathered}
\alpha_{j}(x)=\lambda_{j} \cdot x^{1+\varepsilon}+\mu_{j}, \quad \forall j \in V_{P}, \\
\beta_{k l}(x)=\lambda_{k l} \cdot x^{1+\zeta}, \quad \forall(k, l) \in A,
\end{gathered}
$$

where all coefficients are non-negative. We now solve the problem (14), (15) with specific values of demands $\left\{d_{i}, i \in V_{Q}\right\}$ and coefficients in (17), and evaluate the total cost reduction.

\subsection{Simulation}

Let us assume that consumers have similar demand values, and that the transmission cost in arc $(4,2)$ is higher due to longer distance, while other five arcs have identical coefficients of transmission function (i.e., $\lambda_{42}>\lambda_{a}, \forall a \neq(4,2)$ ). We also assign $\varepsilon=\zeta=0.2$.

We compute and compare minimal total costs of consumers in two different cases: $C_{s i n}$ if each consumer buys energy from a single supplier, and $C_{o p t}$ for a case, when consumers distribute their contracts between several producers.

Table 1. Some of simulation results are given.

\begin{tabular}{ccccccccccc}
\hline$d_{1}$ & $d_{2}$ & $d_{3}$ & $\lambda_{42}$ & $\lambda_{a}$ & $\lambda_{4}, \mu_{4}$ & $\lambda_{5}, \mu_{5}$ & $\lambda_{6}, \mu_{6}$ & $C_{o p t}$ & $C_{\text {sin }}$ & Gain \\
\hline-20 & -22 & -23 & 0.005 & 0.001 & $(0.002,0.2)$ & $(0.001,0.12)$ & $(0.005,0.1)$ & 15.81 & 17.21 & $8.1 \%$ \\
-10 & -12 & -9 & 0.005 & 0.001 & $(0.002,0.2)$ & $(0.001,0.12)$ & $(0.005,0.1)$ & 5.43 & 5.5 & $1.2 \%$ \\
-10 & -12 & -9 & 0.005 & 0.001 & $(0.002,0.2)$ & $(0,0.12)$ & $(0.01,0)$ & 3.98 & 4.33 & $7.9 \%$ \\
\hline
\end{tabular}

It is clear that the cooperative contract distribution reduces the total cost significantly (up to $8 \%$ ). However, this reduction strongly depends on the demand values of consumers and cost functions of producers.

\section{Conclusion and Future Work}

In this work, we described and studied a collaborative model of local area energy consumption in the presence of multiple producers. We showed that the distribution of power flow can be determined by solving an optimization problem based on Kirchhoff's laws, and we formulated the total cost minimization problem considering the congestion in the network arcs. We also considered an example of a network without cycles and presented promising simulation results. There are several possible 
extensions of this model. First of all, it is crucial to consider a dynamic setting over several periods of time (e.g., 24 hours of a day). Second direction is the investigation of cost sharing techniques.

\section{References}

1. Mohsenian-Rad, A.H., Wong, V.W., Jatskevich, J., Schober, R., Leon-Garcia, A.: Autonomous demand-side management based on game-theoretic energy consumption scheduling for the future smart grid. Smart Grid, IEEE Transactions on 1(3), 320--331 (2010)

2. Veit, A., Xu, Y., Zheng, R., Chakraborty, N., Sycara, K.P.: Multiagent coordination for energy consumption scheduling in consumer cooperatives. In: 27th AAAI Conference on Artificial Intelligence, pp. 1362--1368 (2013)

3. Atzeni, I., Ordóňez, L.G., Scutari, G., Palomar, D.P., Fonollosa, J.R.: Demand-side management via distributed energy generation and storage optimization. Smart Grid, IEEE Transactions on 4(2), 866--876 (2013)

4. Atzeni, I., Ordóňez, L.G., Scutari, G., Palomar, D.P., Fonollosa, J.R.: Noncooperative and cooperative optimization of distributed energy generation and storage in the demand-side of the smart grid. Signal Processing, IEEE Transactions on 61(10), 2454--2472 (2013)

5. Alam, M., Ramchurn, S.D., Rogers, A.: Cooperative energy exchange for the efficient use of energy and resources in remote communities. In: Proceedings of the 2013 international conference on Autonomous agents and multi-agent systems, pp. 731--738 (2013)

6. Mihailescu, R.C., Vasirani, M., Ossowski, S.: Dynamic coalition adaptation for efficient agent-based virtual power plants. In: Multiagent System Technologies, pp. 101--112. Springer (2011)

7. Chao, H.P., Peck, S.: A market mechanism for electric power transmission. J. Regulatory Econ. 10(1), 25--59 (1996)

8. O'Neill, R.P., Helman, U., Hobbs, B.F., Stewart, W.R., Rothkopf, M.H.: A joint energy and transmission rights auction: proposal and properties. IEEE Trans. Power Syst. 17(4), 1058-1067 (2002)

9. Dolmatova, M.: Optimal nodal capacity procurement. Advances in Intelligent Systems and Computing 360, 415--423 (2015)

10. Patriksson, M.: The traffic assignment problem: models and methods. VSP, Utrecht, The Netherlands (1994)

11. Zakharov, V.V., Krylatov, A.Y.: Competitive routing of traffic flows by navigation providers. Automation and Remote Control 77(1), 179--189 (2016)

12. Zakharov, V., Krylatov, A.: OD-matrix estimation based on plate scanning. In: Computer Technologies in Physical and Engineering Applications (ICCTPEA), 2014 International Conference on, pp. 209--210 (2014)

13. Bohn, R.E., Caramanis, M.C., Schweppe, F.C.: Optimal pricing in electrical networks over space and time. Rand Journal of Economics 15(3), 360--376 (1984)

14. Bertsekas, D.P.: Nonlinear Programming: 2nd Edition. Athena Scientific, Belmont, Massachusetts (1999)

15. Roth, A.E.: The Shapley value: essays in honor of Lloyd S. Shapley. Cambridge University Press (1988) 